This is an electronic reprint of the original article. This reprint may differ from the original in pagination and typographic detail.

Author(s): Zhang, Di; Chang, Zheng; Yu, Fei Richard; Chen, Xianfu; Hämäläinen, Timo

Title: $\quad$ A Double Auction Mechanism for Virtual Resource Allocation in SDN-based Cellular Network

Year: $\quad 2016$

Version:

Please cite the original version:

Zhang, D., Chang, Z., Yu, F. R., Chen, X., \& Hämäläinen, T. (2016). A Double Auction Mechanism for Virtual Resource Allocation in SDN-based Cellular Network. In Proceedings of IEEE PIMRC 2016 : IEEE 27th International Symposium on Personal, Indoor and Mobile Radio Communications (pp. 1-6). Institute of Electrical and Electronic Engineers. IEEE International Symposium on Personal, Indoor, and Mobile Radio Communications workshops. https://doi.org/10.1109/PIMRC.2016.7794896

All material supplied via JYX is protected by copyright and other intellectual property rights, and duplication or sale of all or part of any of the repository collections is not permitted, except that material may be duplicated by you for your research use or educational purposes in electronic or print form. You must obtain permission for any other use. Electronic or print copies may not be offered, whether for sale or otherwise to anyone who is not an authorised user. 


\title{
A Double Auction Mechanism for Virtual Resource Allocation in SDN-based Cellular Network
}

\author{
Di Zhang*, Zheng Chang*, Fei Richard $\mathrm{Yu}^{\dagger}$, Xianfu Chen ${ }^{\ddagger}$ and Timo Hämäläinen* \\ *Department of Mathematical Information Technology, University of Jyväskylä, P.O.Box 35, FIN-40014 Jyväskylä, Finland \\ ${ }^{\dagger}$ Department of System and Computer Engineering, University of Carleton, Ottawa, ON, Canada \\ $\ddagger$ VTT Technical Research Centre of Finland Ltd, P.O. Box 1100, FIN-90571 Oulu, Finland \\ Email: zhdizhan@student.jyu.fi
}

\begin{abstract}
The explosively growing demands for mobile traffic service bring both challenges and opportunities to wireless networks, among which, wireless network virtualization is proposed as the main evolution towards 5G. In this paper, we first propose a Software Defined Network (SDN) based wireless virtualization architecture for enabling multi-flow transmission in order to save capital expenses (CapEx) and operation expenses (OpEx) significantly with multiple Infrastructures Providers (InPs) and multiple Mobile Virtual Network Operators (MVNOs). We formulate the virtual resource allocation problem with diverse QoS requirements as a social welfare maximization problem with transaction cost. Due to the high computational complexity of formulated problem and hidden information of InPs and MVNOs for SDN controller, we introduce the shadow price for ensuring the desirable economic properties as well as the total welfare of system. Simulations are conducted with different system configurations to show the effectiveness of the proposed SDN based wireless virtualization framework and double auction mechanism.
\end{abstract}

\section{INTRODUCTION}

With tremendous growth of traffic and services in cellular networks, wireless virtualization has been proposed as one of the main evolution trends in the forthcoming fifth generation $(5 \mathrm{G})$ cellular networks [1]. The main idea of wireless virtualization is to decouple the infrastructure from the services it provides, therefore different services can share the same infrastructure, which can further improve resource usage efficiency, as well as reduce the CapEx and OpEx significantly [2]. Although wireless virtualization is a promising technology for next generation networks, many significant research challenges remain to be addressed before its widespread deployment in mobile wireless networks, especially how to design an architecture for encouraging multiple providers to share their infrastructure in order to save CapEx and OpEx [3].

Most existing work for wireless virtualization have taken advantages of game theoretic approaches on resource allocation. Specifically, the authors in [4], [5] and [6] have concentrated their works on applying power-pricing strategies for wireless virtualization, which solve the energy allocation and spectrum sharing problems separately. The cooperative game based spectrum sharing is analysed in [7] and a virtual resource allocation mechanism by using market equilibrium theory is proposed by [8]. The auction mechanisms for dynamic wireless resources (e.g., spectrum, transmission time) have been investigated in [9] and [10]. However, the authors above have only considered merely one Infrastructures Provider ( $I n P)$ in the scenario, which lacks of flexibility for service selection. Besides, the network resources are auctioned without considering the heterogeneous services QoS requirements of User Equipments (UEs).

In this paper, we consider a general network scenario in which multiple InPs (legacy spectrum and infrastructure holders) and multiple MVNOs who provide services to their own subscribers coexist, especially for ensuring the fairness among InPs. InPs attempt to sell their own resources to MVNOs for monetary gains, while MVNOs try to acquire the resource usage permissions from InPs to satisfy own subscribers' communication goals, which generally introduces reward payoffs for InPs. In order to solve the above issues, we consider a double auction mechanism for allocating the physical resource owned by InPs to MVNOs to satisfy the special demands of their subscribers.

While a major confronted challenge is how to allocate the virtualized resource by both considering transmission delay requirement and priority of service offered by the InPs [3]. In order to realize heterogeneous virtualized resource allocation, we introduce SDN controller (SDN is one of the most promising technologies to realize virtual networks and SDN controller operates and manages virtual resource efficiently in response with a global view of network [11]) as the resource allocation control center, which can significantly reduce the signalling delay by decoupling the network to control and data plane. To jointly address the available resource and diversity demands for improving the end-to-end performance, the distinctive features of this paper are as follows:

- We propose a SDN-based wireless virtual resource allocation framework and a double auction mechanism for encouraging both the InPs and MVNOs for joining in order to save CapEx and OpEx significantly.

- The virtual resource allocation problem with diverse QoS requirements is formulated as a social welfare maximization problem with transaction cost.

- Iterative Double Auction (I-DA) algorithms with shadow price are proposed for solving the formulated high computational complexity problem, which ensures that truthful revelation is dominant strategy for both the InPs and MVNOs .

- Simulations are conducted to show the effectiveness of 


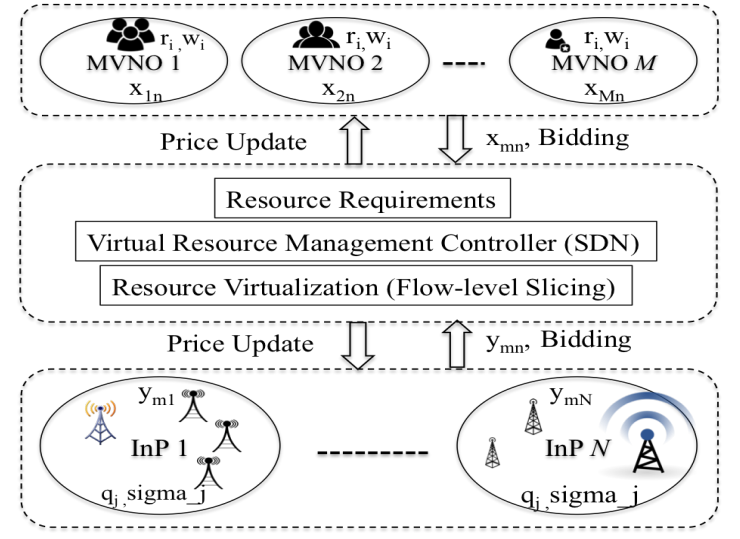

Fig. 1. SDN-based wireless network virtualization framework with multiple InPs and MVNOs

the proposed scheme. It is shown that we can take the advantages of both wireless virtualization and double auction mechanism by considering InPs and MVNOs simultaneously to improve the resource usage efficiency.

\section{SySTEM MODEL}

In this section, we introduce the SDN-based virtual resource allocation framework and the double auction mechanism.

\section{A. Wireless Network Virtualization}

Wireless network virtualization can match resources by dynamically and flexibly slicing the infrastructure and resources into virtual networks to achieve global optimized resource utilization. Three logical roles can be identified after virtualization in Fig.1:

- InPs: they own physical substrate wireless networks, including radio resources (licensed spectrum), base stations (BSs), as well as the core networks and backhauls.

- SDN controller: it is in charge of dividing the service flow into multiple dedicated slices. The virtualized resource is based on flow-level slicing [3] and can enable multi-flow transmission.

- MVNOs: they lease the virtual resource dynamically form the InPs through SDN to satisfy their subscribers' communication requirements.

In this virtualized wireless framework, the MVNOs should pay the usage of physical resource dynamically. To lease physical resources to the MVNOs, the InPs charge fees based on MVNO's usage. MVNOs and InPs have pre-contracts about the price and charging rules.

\section{B. Problem Formulation}

Within one geographical area, we have a set of $\mathcal{N} \triangleq$ $\{1,2, \ldots, N\}$ InPs and a set of $\mathcal{M} \triangleq\{1,2, \ldots, M\}$ MVNOs. Each InP $n \in \mathcal{N}$ has a set of $\mathcal{J}_{n}$ BSs. We denote $\mathcal{J} \triangleq$ $\cup_{n \in \mathcal{N}} \mathcal{J}_{n}=\{1,2, \ldots, J\}$ as the set of all BSs. Each MVNO $m \in \mathcal{M}$ has a set of $\mathcal{I}_{m}$ UEs, where $\mathcal{I} \triangleq \cup_{m \in \mathcal{M}} \mathcal{I}_{m}=$ $\{1,2, \ldots, I\}$ is denoted as the set of all UEs. The wide application of multi-mode terminals guarantees UEs simultaneous access to different RANs, which makes it suitable to access virtualized networks for multi-flow transmission.

The resource slice in this paper is defined as the set of virtual resources (e.g., traffic flows) requested by different MVNOs. We assume the resource slice is based on data rate [2]. By considering the UEs' QoS requirements, we define the UE's demand as the minimum data rate requirements $\left(r_{i, \min }, \forall i \in\right.$ $\mathcal{I}_{m}$ ) with special time-dependency level. Consider the case that each MVNO $m$ would like to require $x_{i j}$ data rate for its UE $i$ through BS $j$, we have $\sum_{j \in \mathcal{J}} x_{i j} \geq r_{i, \min }$. We define the request vector for UE $i$ to all $J$ BSs as: $\mathbf{x}_{i} \triangleq\left(x_{i j}: \forall j \in \mathcal{J}\right)$, and the total request data from MVNO $m$ to $\operatorname{InP} n$ is $X_{m n}=$ $\sum_{i \in \mathcal{I}_{m}} \sum_{j \in \mathcal{J}_{n}} x_{i j}$. The requests for all UEs of each MVNO are given by the matrix $\left(\mathbb{I}_{m}\right.$ is the total number of UEs in MVNO $m$ ):

$$
\mathbf{x}_{m}=\left(\mathbf{x}_{i}: \forall i \in \mathcal{I}_{m}\right), \mathbf{x}_{m} \in \mathcal{C}^{\mathbb{I}_{m} \times N} .
$$

Meanwhile, each BS $j \in \mathcal{J}_{n}$ has total sliced data of $q_{j, \max }$ at time $t$. InP $n \in \mathcal{N}$ want to allocate (sell) $y_{j i} \geq 0$ bytes of data through its $\mathrm{BS} j \in \mathcal{J}_{n}$ to $\mathrm{UE} i \in \mathcal{I}$, and $\sum_{i \in \mathcal{I}} y_{i j} \leq$ $q_{j, \max }$, so the selling vector for each $\operatorname{InP} n$ is $\mathbf{y}_{j} \triangleq\left(y_{i j}\right.$ : $\forall i \in \mathcal{I})$ and the total offered data from InP $n$ to MVNO $m$ is $Y_{m n}=\sum_{i \in \mathcal{I}_{m}} \sum_{j \in \mathcal{J}_{n}} y_{i j}$. The selling matrix for all InPs are given by matrix $\left(\mathbb{J}_{N}\right.$ is the total number of BSs in $\left.\operatorname{InP} n\right)$ :

$$
\mathbf{y}_{n}=\left(\mathbf{y}_{j}: \forall j \in \mathcal{J}_{n}\right), \mathbf{y}_{n} \in \mathcal{C}^{M \times \mathbb{J}_{n}} .
$$

\section{Utility functions}

The concept of utility function is commonly used in microeconomics and refers to the satisfaction level of series actions allocated by decision maker [12] [13]. In this paper, we make use of utility function method for illustrating the business model of both InPs and MVNOs more practically.

For each MVNO $m \in \mathcal{M}$, the packets transmission delay of each flow should be kept low enough to guarantee the QoS requirements requested by its subscribers $i \in \mathcal{I}_{m}$, therefore utility depends on not only the total data rate MVNO offers, but also the time-dependency level. We define the utility function of MVNO $m \in \mathcal{M}$ as $\mathcal{F}_{i}^{m}\left(\omega_{i}, \mathbf{x}_{i}\right)$, where $\omega_{i} \in[0,1]$ is the the weight of UE $i \in \mathcal{I}_{m}$ (dependency level of time). We define $\sum_{i \in \mathcal{I}_{m}} \omega_{i}=1$. Assume that, the utility $\mathcal{F}_{i}^{m}\left(., \mathbf{x}_{i}\right)$ is an increasing, strictly concave and continuously differentiable function of $\mathbf{x}_{i}$ over the range $\mathbf{x}_{i} \geq 0$. The function $\mathcal{F}_{i}^{m}\left(\omega_{i}, x_{i}\right)$ has the characteristics as:

$$
\frac{\partial \mathcal{F}_{i}^{m}\left(\omega_{i}, \mathbf{x}_{i}\right)}{\partial \mathbf{x}_{i}}>0, \frac{\partial \mathcal{F}_{i}^{m}\left(\omega_{i}, \mathbf{x}_{i}\right)}{\partial \omega_{i}}>0 .
$$

The definitions in (3) mean that when the required data rate $\mathbf{x}_{i}$ is the same, the UE $i \in \mathcal{I}_{m}$ who with the higher timedependency weight $\omega_{i}$ can stimulate more profit for MVNO $m$; while when the weights $\omega_{i}$ are the same, the UE who needs higher data $\mathbf{x}_{i}$ can lead more profit for MVNO $m$. This utility function is more practical for measuring the business relationship between operators and subscribers. As the profit of MVNOs are additive, the aggregate utility of MVNO is:

$$
\mathcal{F}_{m}\left(\mathbf{x}_{m}\right)=\sum_{i \in \mathcal{I}_{m}} \mathcal{F}_{i}^{m}\left(\omega_{i}, \mathbf{x}_{i}\right), \forall m \in \mathcal{M}
$$


For each InP $n \in \mathcal{N}$, the objective is to offer as much possibly accepted resource by the MVNOs as possible in order to gain more profit. In virtualized wireless system, we introduce busy level vector: $\sigma_{j} \in(0,1)$ for each BS, which can help balance the system flow allocation. It can be easily obtained under the supervisor of SDN controller. We define function $\Phi_{j}^{n}\left(\sigma_{j}, \mathbf{y}_{j}\right)$ as the utility of each InP $n \in \mathcal{N}$. From reality, we can assume that $\Phi_{j}^{n}\left(\cdot, \mathbf{y}_{j}\right)$ is an positive, increasing and concave function of vector $\mathbf{y}_{j} \geq 0$. So we have the characteristics of function $\Phi_{j}^{n}$ as:

$$
\frac{\partial \Phi_{j}^{n}\left(\sigma_{j}, \mathbf{y}_{j}\right)}{\partial y_{j}}>0, \frac{\partial \Phi_{j}^{n}\left(\sigma_{j}, \mathbf{y}_{j}\right)}{\partial \sigma_{j}}<0 .
$$

The definitions in (5) mean when the allocated data rate is the same, access with higher busy level BS $j \in \mathcal{J}_{n}$ will occur higher cost, which in reverse decrease the profit for each InP $n$; while with the same busy level $\sigma_{j}$, the more data $\mathbf{y}_{i} \mathrm{BS}$ $j \in \mathcal{J}_{n}$ can offer, the more profit InP $n$ can obtain finally. As the utility is additive, for each $\operatorname{InP} n$, the total welfare is:

$$
\Phi_{n}\left(\mathbf{y}_{n}\right)=\sum_{j \in \mathcal{J}_{n}} \Phi_{j}^{n}\left(\sigma_{j}, \mathbf{y}_{j}\right), \forall n \in \mathcal{N} .
$$

\section{Social Welfare Maximization}

SDN controller will act as broker and arrange the whole double-sided auction [4]. In order to ensure the heterogeneity between different InPs and MVNOs, we introduce transaction cost, which includes the costs associated with signalling, backhaul, etc. It is pre-negotiated and when MVNO $m \in \mathcal{M}$ purchases data from InP $n \in \mathcal{N}$, a transaction cost is incurred. Thus, even if two InPs provide the same amount data, they may still be heterogeneous due to these transaction-related costs. The transaction costs are assumed to be common knowledge.

In order to maintain fairness, SDN controller try to find the optimal $\mathbf{x}$ and $\mathbf{y}$ by solving the following social welfare maximization problem:

$$
\begin{aligned}
& \max _{(\mathbf{x}, \mathbf{y})} \sum_{m \in \mathcal{M}} \mathcal{F}_{m}\left(\mathbf{x}_{m}\right)+\sum_{n \in \mathcal{N}} \Phi_{n}\left(\mathbf{y}_{n}\right) \\
&-\sum_{m \in \mathcal{M}} \sum_{n \in \mathcal{N}} p_{m n} T_{m n} . \\
& \text { s.t. } \quad A 1: \sum_{j \in \mathcal{J}} x_{i j} \geq r_{i, \min }, \forall i \in \mathcal{I}, \\
& A 2: \sum_{i \in \mathcal{I}} y_{i j} \leq q_{j, \max }, \forall j \in \mathcal{J}, \\
& A 3: y_{i j} \geq x_{i j}, \forall i \in \mathcal{I}, \forall j \in \mathcal{J}, \\
& A 4: y_{i j} \geq 0, x_{i j} \geq 0, \forall i \in \mathcal{I}, \forall j \in \mathcal{J} .
\end{aligned}
$$

where $p_{m n}>0$ is the pre-negotiated price between MVNO $m$ and InP $n$, which is the cost per unit of data rate. If $m$ and $n$ has no contract, then $p_{m n}=\infty$. $T_{m n}$ is total transmitted data amount between MVNO $m$ and InP $n$, which depends on the final decision variable $\mathbf{x}$ and $\mathbf{y}$. It is obvious that at the equilibrium, $y_{j i}=x_{i j}$ holds $, \forall i \in \mathcal{I}, \forall j \in \mathcal{J}$. So we define:

$$
T_{m n}=\frac{X_{m n}+Y_{m n}}{2}=\frac{\sum_{i \in \mathcal{I}_{m}} \sum_{j \in \mathcal{J}_{n}}\left(x_{i j}+y_{i j}\right)}{2} .
$$

\section{E. Problem Transformation with Shadow Price}

Shadow price is a method for illustrating the marginal utility in constrained optimization in economics [14]. According to properties in (3) and (5), the problem in Section II-D is continuously differentiable, so we can firstly analyse it from its necessary and sufficient Karush-Kuhn-Tucker (KKT) conditions with shadow price $(\boldsymbol{\lambda}, \boldsymbol{\mu}, \boldsymbol{\nu})$ :

$$
\begin{aligned}
\mathcal{L}(\mathbf{x}, \mathbf{y}, \boldsymbol{\lambda}, \boldsymbol{\mu}, \boldsymbol{\nu})= & \max _{(\mathbf{x}, \mathbf{y})} \sum_{m \in \mathcal{M}} \mathcal{F}_{m}\left(\mathbf{x}_{m}\right)+\sum_{n \in \mathcal{N}} \Phi_{n}\left(\mathbf{y}_{n}\right) \\
& -\sum_{m \in \mathcal{M}} \sum_{n \in \mathcal{N}} p_{m n} T_{m n} \\
& -\sum_{i \in \mathcal{I}} \lambda_{i}\left(r_{i, \min }-\sum_{j \in \mathcal{J}} x_{i j}\right) \\
& -\sum_{j \in \mathcal{J}} \mu_{j}\left(\sum_{i \in \mathcal{I}} y_{i j}-q_{j, \max }\right) \\
& -\sum_{i \in \mathcal{I}} \sum_{j \in \mathcal{J}} \nu_{i j}\left(x_{i j}-y_{i j}\right),
\end{aligned}
$$

where $\lambda \triangleq\left(\lambda_{i} \geq 0: \forall i \in \mathcal{I}\right), \boldsymbol{\mu} \triangleq\left(\mu_{j} \geq 0: \forall j \in \mathcal{J}\right)$ are the vector of Lagrangian multipliers corresponding to constraints (8) and (9) separately, and $\boldsymbol{\nu} \triangleq\left(\nu_{i j} \geq 0: \forall i \in \mathcal{I}, \forall j \in \mathcal{J}\right)$ is the matrix of Lagrangian multipliers for constraints (10).

The KKT conditions that yield to the optimal dual variables (shadow price) $\lambda^{*}, \boldsymbol{\mu}^{*}, \boldsymbol{\nu}^{*}$ and optimal primal variables $\mathbf{x}^{*}, \mathbf{y}^{*}$ for problem (13) are given by the following set of equations:

$$
\begin{aligned}
& (B 1): \frac{\partial \mathcal{F}_{i}^{m}\left(\omega_{i}, \mathbf{x}_{i}^{*}\right)}{\partial x_{i j}}=\frac{p_{m n}}{2}-\lambda_{i}^{*}+\nu_{i j}^{*}, \\
& (B 2): \frac{\partial \Phi_{j}^{n}\left(\sigma_{j}, \mathbf{y}_{j}^{*}\right)}{\partial y_{i j}}=\frac{p_{m n}}{2}+\mu_{j}^{*}-\nu_{i j}^{*}, \\
& (B 3): \sum_{j \in \mathcal{J}} x_{i j}^{*}=r_{i, \min }, \\
& (B 4): \sum_{i \in \mathcal{I}} y_{i j}^{*}=q_{j, \max }, \\
& (B 5): x_{i j}^{*}=y_{i j}^{*},
\end{aligned}
$$

where $x_{i j}^{*}, y_{i j}^{*}, \lambda^{*}, \mu^{*}, \nu^{*} \geq 0$ are the global maximizers for the social welfare maximization problem (7).

\section{Iterative Double Auction Algorithms}

We can see that it is infeasible for the SDN controller to derive the optimal SWM solution through solving the problem above directly, due to the limited information of market, especially the valued weights of user priority for MVNO $\omega_{i}\left(i \in \mathcal{I}_{m}\right)$ and utility function for both MVNOs and InPs. Therefore, we take advantage of multi-stage design approach [14] for eliciting the hidden information and Iterative Double Auction (I-DA) algorithms are proposed by first analysing the bidding strategies both from MVNO and InP separately and then the optimal resource allocation problem is solved by SDN controller. 


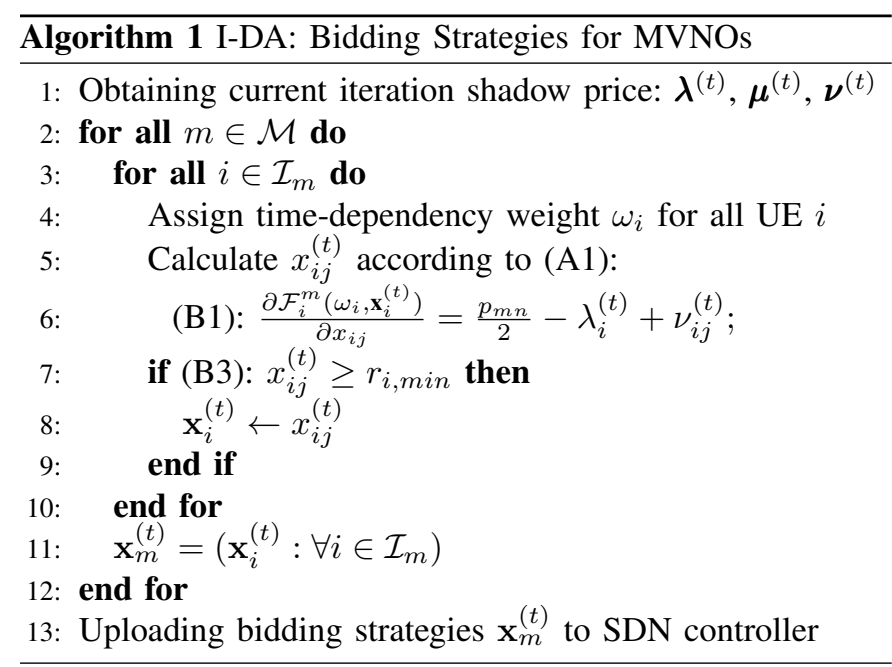

\section{A. The Bidding Strategies}

From the transformed problem in Section II-E, we can see that (B1), (B3), (B5) together combine the optimization bidding strategies for MVNOs, and (B2), (B4), (B5) together combine the optimization bidding strategies for InPs. We introduce the Vickery-Clarke-Groves (VCG) mechanism, which restricts that bidders must follow the truthful valuation for bidding [9]. After obtaining the current iteration shadow prices $\boldsymbol{\lambda}^{(t)}, \boldsymbol{\mu}^{(t)}, \boldsymbol{\nu}^{(t)}$ and assigned weights $\omega_{i}, \sigma_{j}$ of both UEs and BSs, MVNOs and InPs calculating their bidding strategies $\mathbf{x}_{m}, \mathbf{y}_{n}$ separately according to their true valuation of bidding utility: (4) and (6). As the optimal solution is satisfied by solving KKT conditions of primal problem, we solve the utility of both each MVNO $m$ and InP $n$ by (B1) and (B2), which are calculated by the pre-negotiated price $p_{m n}$ between $m$ and $n$ and iteration shadow price $\boldsymbol{\lambda}^{(t)}, \boldsymbol{\mu}^{(t)}, \boldsymbol{\nu}^{(t)}$ produced by constraints (8) and (9). We can see the details in Algorithm 1 and Algorithm 2.

\section{B. Evaluating Dual Variables}

We define the dual objective $g(\boldsymbol{\lambda}, \boldsymbol{\mu}, \boldsymbol{\nu})$ as an unconstrained maximization of Lagrangian (13):

$$
g(\boldsymbol{\lambda}, \boldsymbol{\mu}, \boldsymbol{\nu})=\max _{\mathbf{x}, \mathbf{y}} \mathcal{L}(\mathbf{x}, \mathbf{y}, \boldsymbol{\lambda}, \boldsymbol{\mu}, \boldsymbol{\nu}) .
$$

The dual optimization problem is:

$$
\begin{aligned}
& \min g(\boldsymbol{\lambda}, \boldsymbol{\mu}, \boldsymbol{\nu}), \\
& \text { s.t. } \boldsymbol{\lambda}, \boldsymbol{\mu}, \boldsymbol{\nu} \geq 0 .
\end{aligned}
$$

As the objective function in (13) is strictly concave and the constraint is compact and convex, the results guarantee that the primal problem (7) and dual problem (16) have the same solution, i.e. the duality gap between primal and dual function can be assumed negligible. Consequently, we can update the dual variables $(\boldsymbol{\lambda}, \boldsymbol{\mu}, \boldsymbol{\nu})$ by using a sub-gradient
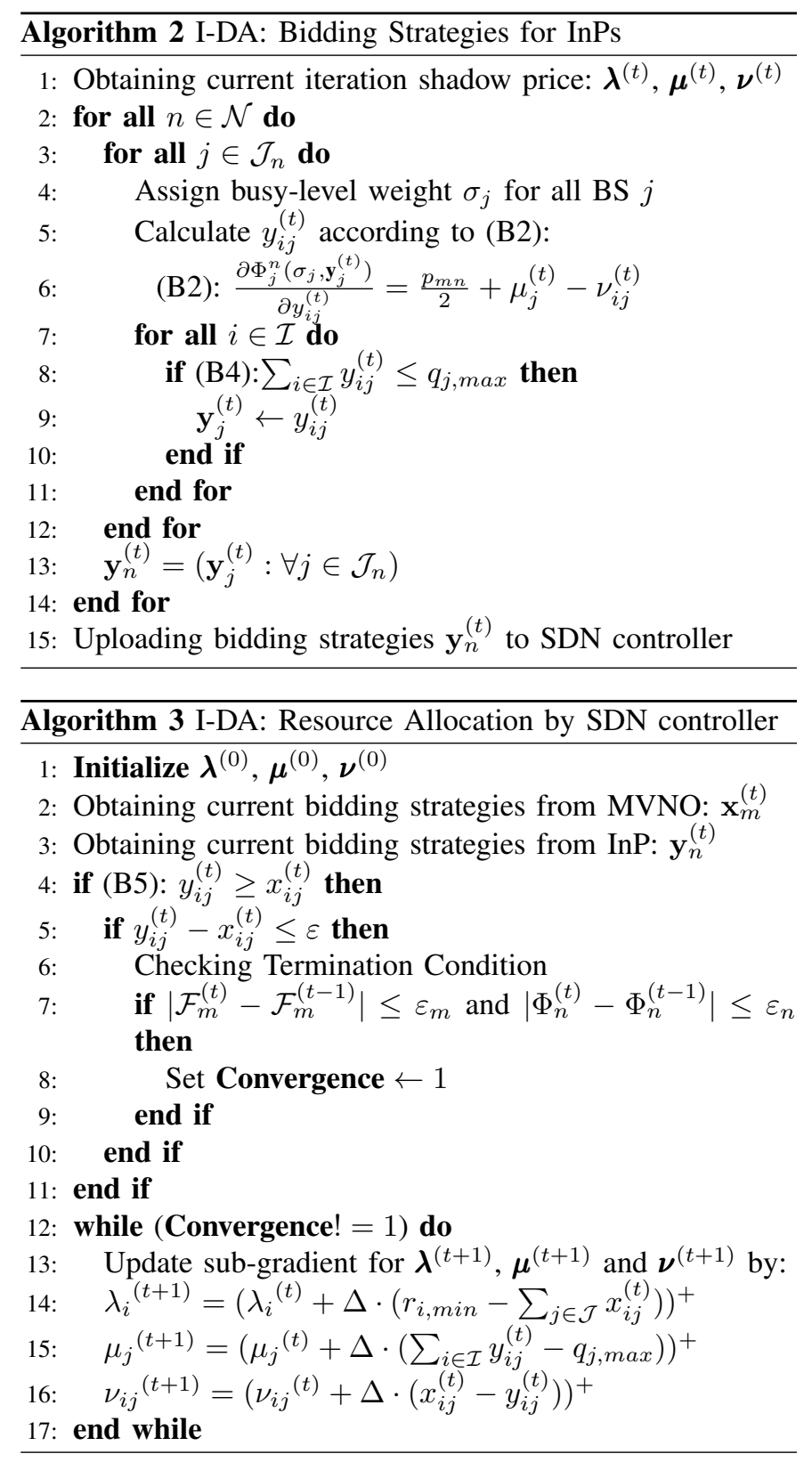

descent method:

$$
\begin{aligned}
& \lambda_{i}^{(t+1)}=\left(\lambda_{i}^{(t)}-\Delta \lambda \frac{\partial \mathcal{L}(\cdot)}{\partial \lambda_{i}}\right)^{+}, \forall i \in \mathcal{I}, \\
& \mu_{j}^{(t+1)}=\left(\mu_{j}^{(t)}-\Delta \mu \frac{\partial \mathcal{L}(\cdot)}{\partial \mu_{j}}\right)^{+}, \forall j \in \mathcal{J}, \\
& \nu_{i j}^{(t+1)}=\left(\nu_{i j}^{(t)}-\Delta \nu \frac{\partial \mathcal{L}(\cdot)}{\partial \nu_{i j}}\right)^{+}, \forall i \in \mathcal{I}, \forall j \in \mathcal{J} .
\end{aligned}
$$

where ()$^{+}$denotes the projection onto the non-negative orthant and ensures that feasibility constraints $\lambda_{i}^{(t+1)} \geq 0, \mu_{j}^{(t+1)} \geq 0$ and $\nu_{i j}^{(t+1)} \geq 0$. In order to simplify the overall parameter setting, we set here the $\Delta \lambda=\Delta \mu=\Delta \nu=\Delta$.

Meanwhile, we define $\left(\varepsilon_{m}, \varepsilon_{n}, \varepsilon\right)$ as the convergence vector. If the calculating bidding strategies satisfy the convergence vector, the I-DA algorithms terminate, otherwise, the 


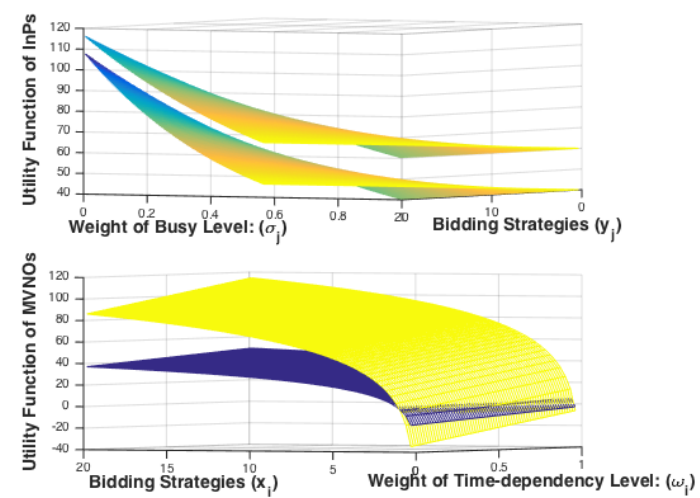

Fig. 2. The monotonicity of utility functions of InPs and MVNOs separately. Where $\beta_{n}=[20,30]$ and $\alpha_{m}=[\ln (2),(\ln 5)]$

I-DA algorithms continue.

\section{Performance Evaluation}

In this section, we provide the simulation results to illustrate theoretical analysis and performance of the proposed I-DA algorithm. We consider the scenario that there are two InPs and two MVNOs operate within the same geographical area. They are all controlled by one SDN controller and the system resources are sliced in the flow-level and can be specified as "data rate". The utility functions of both MVNOs and InPs are respectively defined as:

$$
\begin{aligned}
& \mathcal{F}_{m}=20 \cdot \sum_{i \in \mathcal{I}_{m}} \log _{\alpha_{m}}\left(e^{\omega_{i}} \cdot \mathbf{x}_{i}\right), m \in\{1,2\} \\
& \Phi_{n}=2 \cdot \beta_{n} \sum_{j \in \mathcal{J}_{n}} e^{\frac{\left(1-\sigma_{j}\right) \cdot \mathbf{y}_{j}}{\beta_{n}}}, n \in\{1,2\}
\end{aligned}
$$

Through Fig. 2 we can see that, the setting is satisfied the monotonicity properties defined in (3) and (5). We suppose that there are 5 UEs that active with minimum data rate requirement $r_{i, \text { min }}=10(\mathrm{bit} / \mathrm{s} / \mathrm{Hz})$ during time-slot $t$. The first 3 belongs to MVNO1 and the last 2 belong to MVNO2. There are 3 BSs totally in the geographical area and at $t$, they all have maximum available data rate $q_{j, \max }=20(\mathrm{bit} / \mathrm{s} / \mathrm{Hz})$. The first 2 belongs to InP1, and the last 1 belongs to InP2. MVNOs assign the UEs' weight $\omega_{i}$ according to their time-dependency level as $[0.5,0.3,0.2]$ and $[0.6,0.4]$. Meanwhile, InPs assign the BS's weight $\sigma_{j}$ according to their busy level as $[0.5,0.3]$ and $[0.6]$. We assume the pre-negotiated transaction cost vector is $p_{m n}=[2,3 ; 3,2]$.

Fig. 3 shows the convergence of proposed I-DA algorithm and the effect of step size $\Delta$ in Algorithm 3. As shown in this figure, the gap between the I-DA algorithms and global optimal value is narrow, meanwhile it is absolutely larger than the random allocation value. This means the effectiveness of I-DA algorithms is equivalent to the optimal in terms of the overall utility. It can be found that the results with different $\Delta$ finally converge to almost the same utility value with only a small gap. However, the value of $\Delta$ affects the rate of

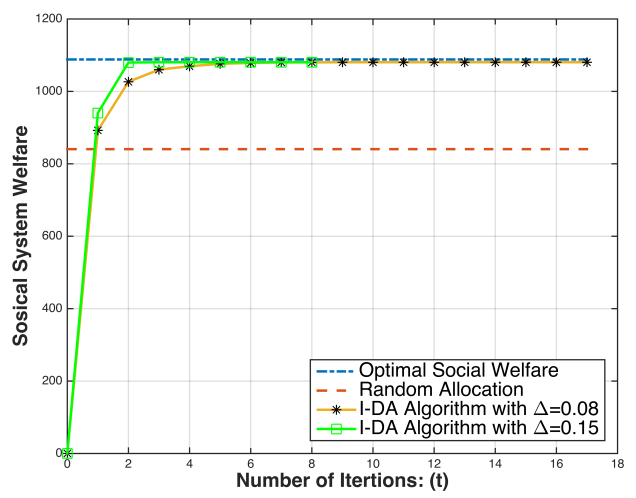

Fig. 3. The convergence process of I-DA and the effect of $\Delta$
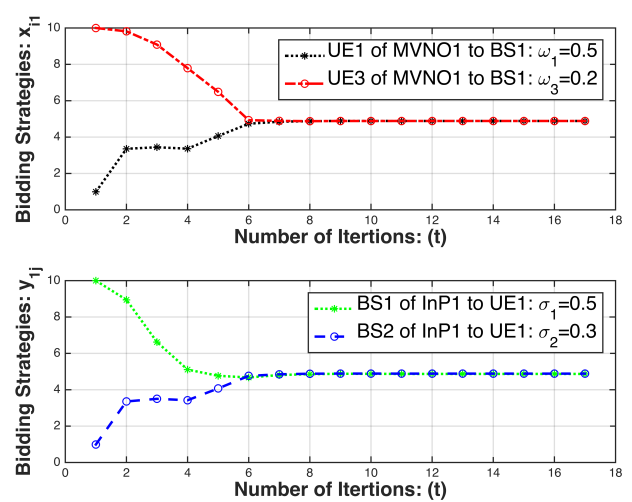

Fig. 4. The impact of UE's time-dependency $\omega_{i}$ and BS's busy-Level $\sigma_{j}$ for bidding strategies $x_{i j}, y_{i j}$

convergence. $\Delta=0.15$ gives higher convergence rate than $\Delta=0.08$. Nevertheless, if we further increase the step, we will see that the I-DA algorithm does not converge to the optimal solution.

Fig. 4 illustrates the impact of QoS requirements $\omega_{i}$ and $\sigma_{j}$ in the utility function for MVNO and InPs' bidding strategies iteration by iteration separately. For MVNOs, we take the bid evolution of UE1 and UE3 (belonged to MVNO1) to BS1 (belonged to InP1) as reference. As shown in this figure, MVNO1 changes the bidding strategies according to the time-dependency weight $\omega_{i}$, the one with higher emergency service requirement (higher weight) has higher priority when bidding. The MVNO will increase the bids value for that UE accordingly and meanwhile decrease the bid value for UE who has less priority. We can see the same phenomenon for InPs, where BS2 with less busy level $\left(\sigma_{2}=0.3\right)$ has higher priority than BS1 who has higher busy level $\left(\sigma_{1}=0.5\right)$ when bidding. The reason is that, the InP can increase its own payoff by either increase the bidding value $\left(y_{i j}\right)$ for less busy BS, or decrease the bidding value for higher occupied BS. The phenomenon also proves that MVNOs and InPs bid strictly and truthfully according to their utility function (defined in (3) and (5)) in I-DA algorithms. 

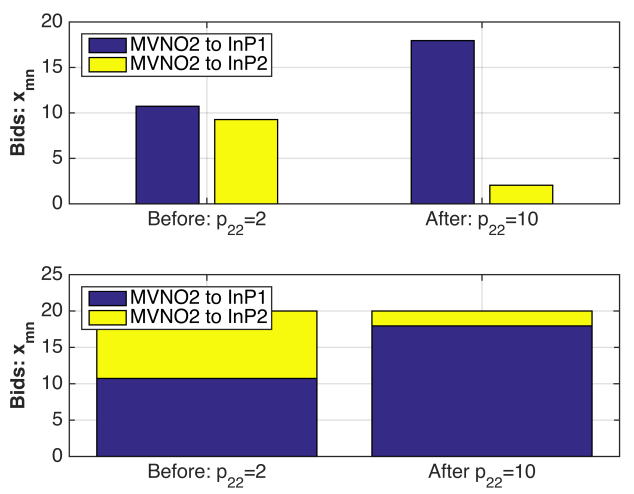

Fig. 5. Evolution of bidding with transaction cost $p_{m n}$ increasing

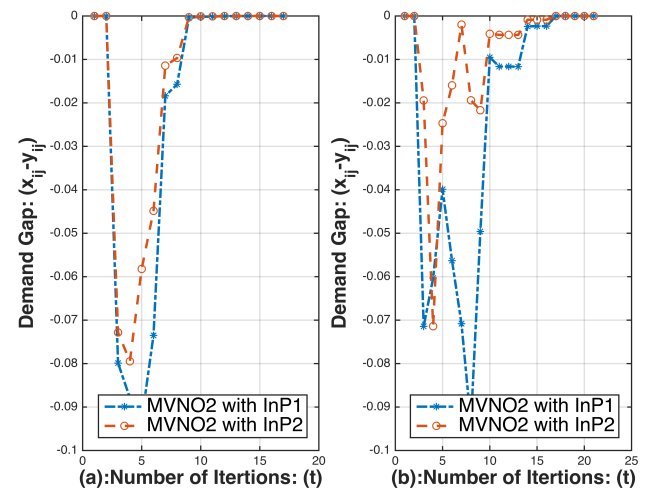

Fig. 6. Evolution of bidding gap with transaction cost $p_{m n}$ increasing

In Figs. 5 and 6, we evaluate the effect of transaction cost $p_{m n}$ on bidding strategies evolution. We take the bidding of $\mathrm{MVNO} 2$ to $\mathrm{InP} 1$ and $\mathrm{InP} 2$ as reference. As shown in Fig. 5, the transaction cost between $\mathrm{MVNO} 2$ and InP2 is increased from $p_{22}=2$ per unit data rate to $p_{22}=10$. Even though the final total bidding value are the same (according to UEs' demand), the bidding strategies to InP1 and InP 2 change significantly. MVNO2 evolves by decreasing bidding value from $8(\mathrm{bit} / \mathrm{s} / \mathrm{Hz})$ to $2(\mathrm{bit} / \mathrm{s} / \mathrm{Hz})$ to ensure higher utility. Besides, in Fig. 6, we can see that, at the beginning, the bidding strategies of MVNO2 are nearly the same, especially before 5 -th iteration. While after re-negotiate the transaction cost, $\left(p_{22}=2 \rightarrow 10\right)$, the MVNO2, as well as InP1 and InP2 evolve the bidding strategies significantly, especially between 5th and 10th iteration. The reason behind the behaviour is that both MVNOs and InPs want to ensure the utility during bidding (see Algorithm 1, Line 6 and Algorithm 2, Line 6).

Fig. 6 also presents the convergence of $\mathbf{x}_{i}$ and $\mathbf{y}_{j}$. Specially, we see that the gap between the requested demand and offered traffic gradually converges to zero, which satisfies the condition B5 in (14). This means that the MVNOs and InPs agree on the amount of data and negotiated transaction cost $p_{m n}$, as well as the SDN controller's central control.

\section{CONCLUSIONS}

In this paper, we investigated a SDN-based architecture for attracting multiple InPs and MVNOs sharing the virtual resource allocation in order to reduce CapEx and OpEx. We first virtualized the physical resources as virtual flow-level resources. After virtualization, MVNOs can help their own subscribers access to different InPs to get performance gain. Furthermore, we formulated the virtual resource allocation problem as an optimization problem by maximizing the total utility of system. In order to solve it efficiently, the virtual resource allocation problem is transformed to an iterative double auction problem with transaction cost. In this process, the MVNOs and InPs bid gradually according to their own utility function, as well as the shadow price introduced by SDN controller until the system converges. Simulation results also demonstrated the effectiveness and good convergence performance of our proposed I-DA algorithm. Future work in progress is to consider more realistic simulations with SDN, as well as the dynamic traffic requirement of UEs in the proposed SDN based virtualization architecture.

\section{ACKNOWLEDGMENT}

The author would like to thank Chinese Scholarship Council (201306080004) and HPY Foundation for supporting.

\section{REFERENCES}

[1] C. Liang and F. R. Yu, "Wireless network virtualization: A survey, some research issues and challenges," IEEE Commun. Surv. Tut., vol. 17, no. 1, pp. 358-380, Firstquarter 2015.

[2] E. Hossain and M. Hasan, "5g cellular: key enabling technologies and research challenges," IEEE Instrum. Meas. Mag., vol. 18, no. 3, pp. 11-21, June 2015.

[3] Z. Feng and et al, "An effective approach to $5 \mathrm{~g}$ : Wireless network virtualization,' IEEE Commun. Mag., vol. 53, no. 12, pp. 53-59, 2015.

[4] D. Zhang, Z. Chang, and T. Hämäläinen, "Reverse combinatorial auction based resource allocation in heterogeneous software defined network with infrastructure sharing," in Proc. IEEE/VTC-Spring'16, Nanjing, May 2016, to be published.

[5] D. Zhang and et al, "Energy efficient resource allocation in heterogenous software defined network : A reverse combinatorial auction approach," in Proc. IEEE/CIC ICCC'15, ShenZhen, Nov 2015, pp. 739-744.

[6] B. Cao and et al, "Power allocation in wireless network virtualization with buyer/seller and auction game," in Proc. IEEE/GLOBECOM'15, San Diego, CA, Dec 2015, pp. 1-6.

[7] B. Liu and H. Tian, "A bankruptcy game-based resource allocation approach among virtual mobile operators," IEEE Commun. Lett., vol. 17, no. 7, pp. 1420-1423, July 2013.

[8] G. Zhang and et al, "Virtual resource allocation for wireless virtualization networks using market equilibrium theory," in Proc. IEEE/INFOCOM'15, Hong Kong, April 2015, pp. 366-371.

[9] K. Zhu and E. Hossain, "Virtualization of $5 \mathrm{~g}$ cellular networks as a hierarchical combinatorial auction," IEEE Trans. Mobile Comput., 2015, to be published.

[10] F. Fu and U. C. Kozat, "Stochastic game for wireless network virtualization," IEEE/ACM Trans. Netw., vol. 21, no. 1, pp. 84-97, Feb 2013.

[11] M. Yang and et al, "Software-defined and virtualized future mobile and wireless networks: A survey," Mobile Networks and Applications, vol. 20, no. 1, pp. 4-18, 2015.

[12] G. Iosifidis and et al, "A double-auction mechanism for mobile dataoffloading markets," IEEE/ACM Trans. Netw., vol. 23, no. 5, pp. 16341647, Oct 2015.

[13] C. Liang and et al, "Virtual resource allocation in information-centric wireless virtual networks," IEEE Trans. Veh. Technol, 2016, to be published.

[14] L. Y. Chu and Z.-J. M. Shen, "Truthful double auction mechanisms," Operations research, vol. 56, no. 1, pp. 102-120, 2008. 